\title{
Non-esterified fatty acids and the liver: why is insulin secreted into the portal vein?
}

\author{
R.N. Bergman \\ Department of Physiology and Biophysics, Keck School of Medicine, University of Southern California, Los Angeles, Calif. USA
}

\section{Abstract}

Aims/hypothesis. Insulin control of glucose output is a major mechanism by which appropriate amounts of glucose are produced to supply energy to the central nervous system, without causing long-term increases of the plasma glucose concentration. It is hypothesised that the primary route by which insulin maintains control over glucose production is indirect and is mediated by regulation of non-esterified fatty acid release from the adipocyte. The question arises as to why evolution has chosen insulin to be secreted into the portal vein, if control of the liver is partially or primarily indirect. It is suggested that alterations in hepatic insulin clearance which attend increases in central adiposity are an important part of the compensation for insulin resistance and limit the necessity for up-regulation of insulin secretion in insulin resistance secondary to central adiposity.

Methods. Review of research from author's group and other laboratories.

Results. Data over the previous decade indicate that suppression of glucose output by increased insulin is a relatively slow process, much slower than the rate of binding of insulin to hepatocytes. One explanation is that insulin acts on an extrahepatic tissue, which in turn alters a signal to the liver, reducing glucose output. Additional evidence for an extrahepatic primary effect of insulin emerges from experiments in which insulin was given portally or peripherally at half the portal dose. Endogenous glucose production was related to systemic, not portal insulin, supporting the concept that the primary step in insulin's action on liver is on some other tissue, altering signalling to the liver itself. Strong correlation between plasma non-esterified fatty acids (NEFA) and liver glucose output suggests that the primary effect is on the adipocyte. The primacy of the adipocyte locus for the insulin effect included data that insulin's action on liver is prevented when plasma NEFA are maintained, as well as data showing proportional decline in glucose production and fatty acids when antilipolysis is induced by an adenosine agonist.

Why then, from an evolutionary point of view is insulin secreted into the portal vein? Institution of central adiposity in dogs with fat feeding causes hepatic insulin resistance, at least partially due to the provision of NEFA in portal blood. The initial response to resistance is enhanced beta-cell sensitivity to glucose; a secondary compensation is, however, a substantial reduction in liver clearance, allowing for a greater proportion of secreted insulin to reach muscle, where it can more efficiently stimulate glucose utilisation.

Conclusion/interpretation. Non-esterified fatty acids act as a signal as well as a metabolic substrate. They can regulate glucose utilisation in muscle and apparently are important signals to the liver and the beta cells as well. The importance of portal vein NEFA concentrations to the function of the liver could explain insulin resistance of the liver with central pattern obesity. [Diabetologia (2000) 43: 946-952]

Keywords Adipocyte, liver, gluconeogenesis, central adiposity, single gateway hypothesis. 
Non-esterified fatty acids and insulin resistance

Insulin resistance is a primary risk factor for Type II (non-insulin-dependent) diabetes mellitus [1]. It is also associated with cardiovascular disease, hypertension and certain forms of cancer [2-4]. It has been identified as a central feature in clusters of high-risk conditions in the so-called metabolic syndrome, or "syndrome $x$ " [5]. New pharmacological agents which reduce insulin resistance such as the thiazolidinediones also improve glucose control in patients with Type II diabetes and could help to prevent the disease $[6,7]$. Recent evidence supports the concept that a major palliative effect of thiazolidinediones is to reduce blood-borne fatty acids ("keep fatty acids in the adipocyte") [8]. Thus, it appears that control of NEFA concentrations in the blood could be an important approach to reduce insulin resistance and the complications of the insulin resistant state [9].

To optimise therapy with insulin sensitising agents, it would be useful to understand how NEFA contribute to insulin resistance. Although our understanding of the relation between NEFA and insulin action remains primitive, recent developments regarding how NEFA interact with carbohydrate metabolism have been illuminating. After carbohydrate ingestion, insulin is secreted and the hormone acts to stimulate glucose utilisation and suppress endogenous glucose production (EGP) by liver (and kidney). There is concomitant suppression of lipolysis and concomitant reduction in plasma NEFA. An increase in NEFA can considerably inhibit skeletal muscle glucose utilisation, possibly by interfering with insulin signalling and glucose transport, or phosphorylation or both [10]. Reduction in NEFA could play some part in the enhancement of glucose disposal [11] (and muscle glycogen synthesis [10]) after food intake. The relation between insulin, NEFA reduction and suppression of EGP is more obscure.

\section{Insulin and glucose production}

Recent developments in our laboratory and others have shed some light on the complex relation among insulin, NEFA and glucose production. The traditional view has been that following carbohydrate intake, insulin is secreted by the beta cells, increasing portal insulin concentrations and resulting in direct suppression of liver glucose production [12]. It seems logical that insulin should play this important part because it is secreted directly into the liver. By analogy with the cerebral portal system, it can be supposed that the beta-cell/hepatocyte communication link provides exquisite and appropriate moment-to-moment provision of glucose in response to need. Recent evidence has, however, sharply questioned this traditional view of the way EGP is controlled by insulin.

Tracer dilution methods have clearly shown that an increase in insulin concentration by peripheral infusion causes suppression of EGP, even if plasma glu- cose concentrations are clamped at basal [13]. It has, however, been difficult to reproduce this fundamental relation in liver or liver cells in vitro, except under hyperglucagonaemic conditions [14, 15]. Although this failure has been assigned to lack of proper oxygenation or lack of intact neural control in vitro, it was suggested years ago that insulin regulation of liver glucose output is mediated by an extrahepatic factor [16]. This suggestion was recapitulated more recently by a flurry of evidence showing a sluggish relation between changing portal insulin concentration and suppression of EGP under a variety of conditions in vivo [17-19]. Although evidence obtained in vivo had suggested that suppression of EGP after insulin treatment was rapid [20], several groups showed that this perception resulted from artefacts in tracer dilution methodology [21-23]. Resolution of these artefacts, which were due to modelling error [24] as well as tracer contaminants [25], made it possible to obtain accurate assessment of the rate of suppression of liver glucose production due to insulin. For example, in our laboratory we showed that insulin acts rather slowly in vivo to suppress EGP [17] and this result has been confirmed in vivo in human subjects [26]. Slow kinetics of insulin action on liver are in stark contrast to binding of insulin to liver receptors, which is almost immediate under portal hyperinsulinaemic conditions [27]. The lethargic kinetics of EGP suppression is reminiscent of the sluggish stimulation of glucose uptake by muscle [20]. In the dog model, under conditions of basal glucose and glucagon, insulin stimulation of glucose disposal and insulin suppression of endogenous glucose output had similar time courses, with half-times of about $45 \mathrm{~min}$. This was in sharp contrast to the effects of insulin on glucose uptake in vitro, which progressed with a halftime of approximately $10 \mathrm{~min}$.

Measurement of insulin in interstitial fluid (or in lymph, which is reflective of interstitial fluid) has led to the concept that the sluggish effects of insulin on glucose uptake in vivo are due to retarded transport of the hormone across the endothelial barrier of skeletal muscle [28]. This simple concept does not explain why insulin acts slowly on liver: liver capillaries are highly fenestrated and portal vein insulin rapidly binds to liver receptors, yet EGP declines slowly when plasma insulin concentration is increased. Either the post-receptor action of insulin on the liver itself is a slow process or insulin must cross the endothelial barrier of another tissue and generate a blood-borne signal ("second signal") which in turn suppresses the liver. Recent studies from our laboratory [19, 29-31] and others [32-36] have supported the latter concept; insulin's effect to suppress glucose output is mediated at least in part by an extrahepatic tissue. We have referred to this phenomenon as the "single gateway hypothesis" [28] (Fig.1). The endothelial barrier to insulin transport across non-hepatic 


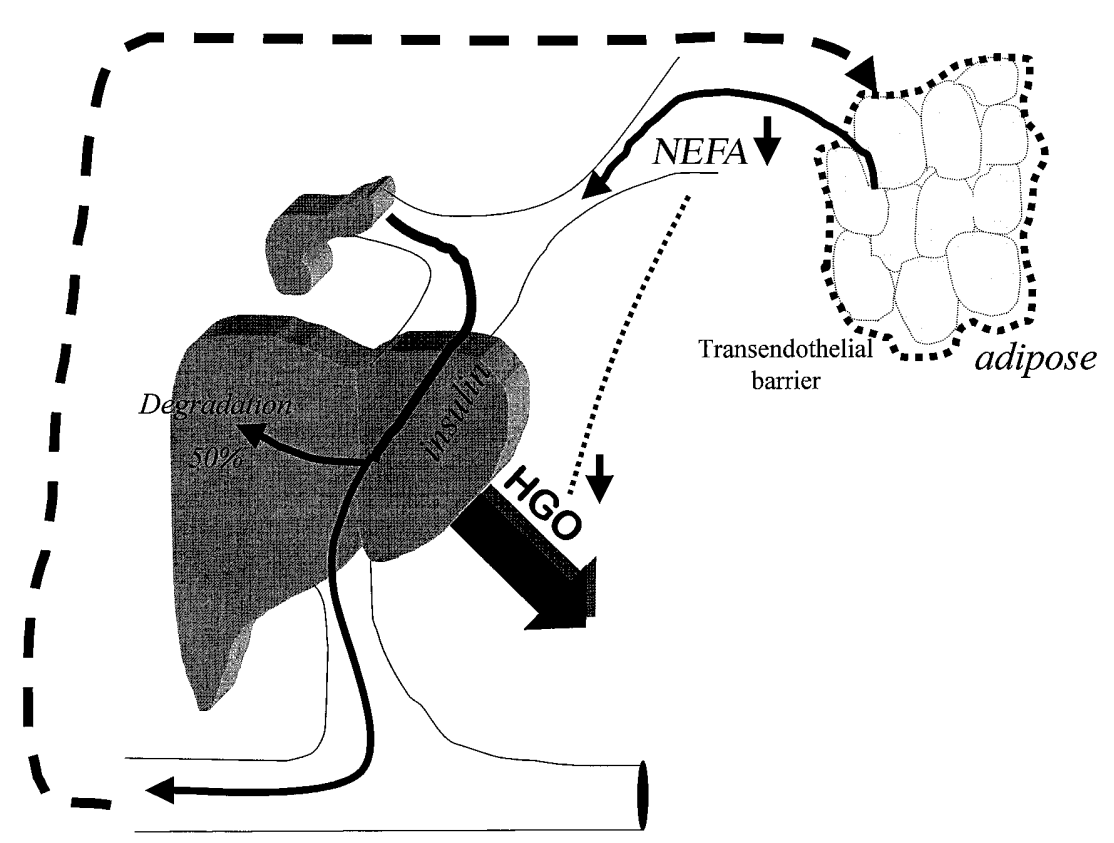

Fig. 1. The single gateway hypothesis. Insulin is secreted by the pancreas, enters the portal vein and approximately $50 \%$ is degraded by liver. The remaining insulin survives hepatic passage and enters the systemic circulation. Insulin crosses the endothelial barrier of adipose tissue slowly, resulting in a slow reduction in plasma non-esterified fatty acids ("second signal"). This slow reduction of NEFA is envisioned to be a primary reason for the ultimate reduction in hepatic glucose production (HGO). This hypothesis can account for (1) the sluggish effect of insulin to reduce liver glucose output and (2) the strong dynamic and steady-state relation between plasma NEFA and endogenous glucose production

insulin sensitive tissues (muscle and adipose) limits in time not only the effect of insulin to enhance glucose uptake but also the effect to suppress EGP.

The "second" signal

The search for the extrahepatic "second signal" exploited a protocol introduced in our laboratory some years ago: we compared the effect of intraportal insulin infusions in conscious dogs, with control experiments in which insulin was infused peripherally at half the intraportal infusion rate (Fig.2) [29]. Due to approximately $50 \%$ first pass hepatic extraction of insulin, these protocols result in matched systemic insulin concentrations but very different portal insulin concentrations. Intraportal insulin infusions compared with half-rate systemic insulin infusions had equipotent effects on suppressing EGP, despite the very different portal insulin concentrations. Suppression of EGP also closely matched the time course of peripheral rather than portal insulin concentrations. Repetition [19] of the half-dose protocol enabled the search for the elusive extrahepatic signal which mediates insulin's suppression of liver glucose production: the only signal we found which correlated strongly with suppression of EGP was plasma NEFA [19, 30]. Thus, we suggested that much of the effect of insulin to control liver glucose production is mediated by plasma NEFA. The single gateway concept was thus modified to suggest that insulin crosses the endothelial barrier at the adipocyte slowly; ultimately adipocyte hormone sensitive lipase is inhibited and liver (and kidney?) glucose production is reduced. Of course, the concept that NEFA can alter glucose production is far from new: NEFA have long been known to stimulate gluconeogenesis [37]. What is new about the single gateway concept is the introduction of NEFA as a moment-to-moment controller of (i. e. a "signal" to) EGP and the replacement of insulin itself by NEFA as the primary regulator of glucose output by the liver.

The half-dose protocol has been adopted and modified to good effect by another group who confirmed that extrahepatic effects are extremely important in the regulation of glucose output by the liver $[32,33,36]$. They have shown that the extrahepatic effect of insulin is dominant for suppression of EGP in the depancreatised dog as well as Type II diabetic patients $[32,36]$, although they have emphasised that a direct effect of insulin has a partial role in regulating EGP in normal subjects [33]. Additionally, they confirmed the potential importance of NEFA as a mediator of the extrahepatic (i.e. "indirect") role of insulin to control EGP [34] and their results support the single gateway concept regarding the kinetics of insulin's effect on EGP. Others have also emphasised the potential role of insulin suppression of the glucagon effect on liver glucose output as having a partial role in the indirect effect of insulin [35]. Most of the studies from our laboratory were done under conditions of constant glucagon 

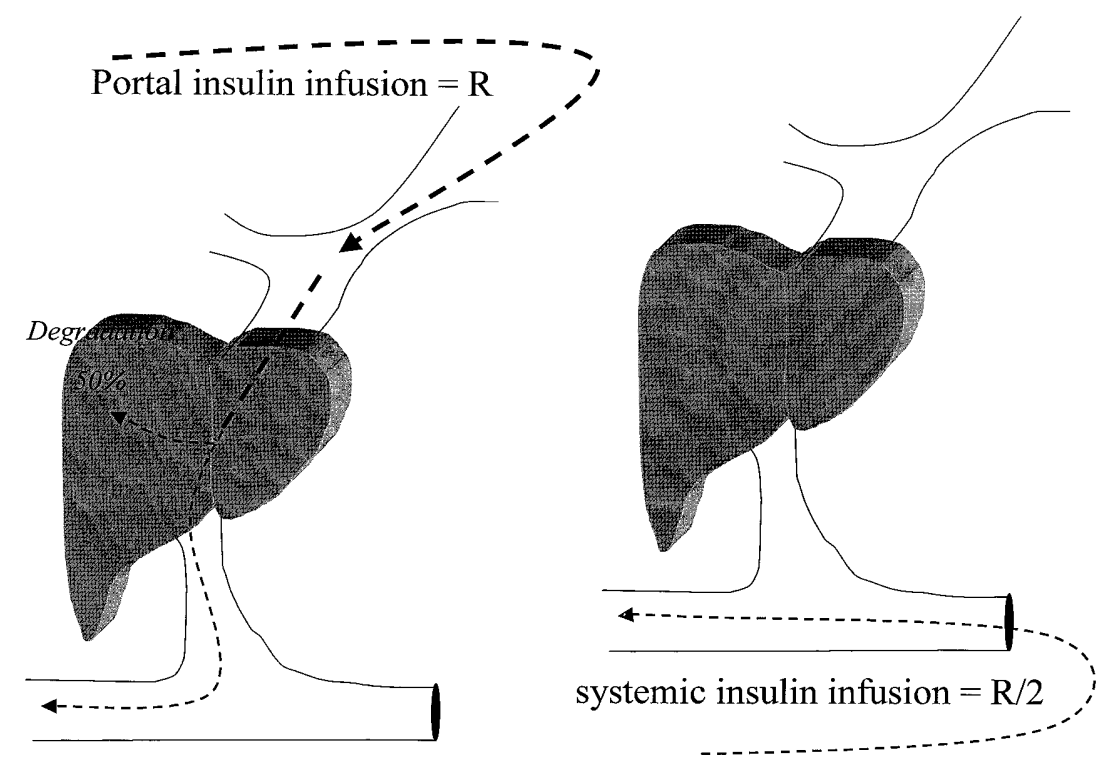

Fig. 2 A, B. In one set of experiments (A), insulin is infused into the portal vein at one of several doses (denoted ' $R$ '), suppressing liver glucose output (approximately half the infused insulin will reach the systemic circulation and about half will be degraded by the liver on first pass). In a companion set of experiments $(\mathbf{B})$, insulin is infused into the systemic circulation at half the rates as in the initial protocol (i.e. 'R/2'). The companion experiments are designed to yield matched systemic insulin concentrations but different portal concentrations [29]

and would therefore not show any effect of insulininduced changes in glucagon on glucose output. The role of the extrahepatic effect of insulin at several rates of glucagon replacement has, however, been quantified with the conclusion that only under hyperglucagonaemic conditions does insulin inhibition of the glucagon effect have more than a minor role in determination of liver glucose production [31].

It has been important to devise new methods to carefully integrate the many factors which can impinge on EGP (insulin itself, glucagon, NEFA, gluconeogenic precursors). Among these are not only the half-portal infusion method (Fig. 2) but also the tolbutamide infusion technique [33]. Each of these methods has limitations. For example, for one protocol [29], it is necessary to know the first-pass extraction of insulin by liver. This has been assumed to be $50 \%$; in dogs $57 \%$ of portally appearing insulin is, however, degraded by liver [19]. Not having a very accurate measure of hepatic insulin clearance before the experiment can on the one hand lead to imprecision in matching systemic insulin concentrations when comparing the portal with the peripheral infusion rates and possible misinterpretation of results. On the other hand, calculation of portal insulin appearance using deconvolution can use assumed Cpeptide clearance variables but these are not accurate for all patient groups [38]. Finally, it is of extreme importance to control the hepatic concentration of glucose itself. Glucose is a powerful regulator of liver glucose output and uptake [39-41] and therefore experimental approaches in which glucose is not well controlled can yield conflicting results.

Additional evidence that NEFA are the primary signal to liver include data that infusion of lipid emulsion during hyperinsulinaemia blocks suppression of endogenous glucose production [30] and recent data that suppression of lipolysis with an adenosine receptor agonist lowers NEFA and glucose production without changing plasma insulin [42].

Although further examination of the relative importance of insulin control of the adipocyte compared with interaction with glucagon's effects on liver is justified, it seems: (1) the indirect, extrahepatic effect of insulin is of major importance in control of endogenous glucose production, (2) insulin control of lipolysis is probably a primary, if not the only, mechanism by which the indirect effect on the liver is mediated and (3) under hyperglucagonaemic conditions (stress, diabetes, long-term fasting) the classic interaction between insulin and glucagon could have a more important role in regulation of EGP.

\section{Mechanism of control by NEFA}

The mechanism(s) by which NEFA control the liver is still not known. That NEFA can increase gluconeogenesis has been known for many years and it is not unreasonable to suppose that this is one, if not the only, mechanism by which the extrahepatic effect is mediated. Treating humans with nicotinic acid resulted in a decrease in plasma NEFA, followed by a postdrug rebound and overshoot in NEFA concentrations [43]. This pattern caused first a decline and then a rebound rise of gluconeogenesis. Of great interest is that glycogenolysis was regulated conversely to gluconeogenesis such that NEFA did not seem to alter 
EGP despite wide fluctuation in the gluconeogenic rate. Glucose was not controlled in these experiments (it was reduced about $0.8 \mathrm{mmol} / \mathrm{l}$ by nicotinic acid infusion). Possibly the decrement in liver glucose (or intrahepatic glucose-6-phosphate) up-regulated glycogen breakdown. Increased glycogen degradation could then compensate for the reduction in gluconeogenesis and result in unchanged liver glucose production.

It is also possible that NEFA play a part in moment-to-moment regulation of liver glucose output by a mechanism other than gluconeogenic regulation. Whether NEFA regulate glycogen metabolism in liver (as they do in muscle) is not known. Even so, it is of interest to ask "what is the explanation for the exquisite reciprocal control of glycogenolysis compared with gluconeogenesis" [43-45]? Induction of glucose6-phosphatase by NEFA has been reported [46]. Is it possible that NEFA control glucose-6-phosphatase on a moment-to-moment basis? Such a mechanism would explain why total hepatic glucose output could be appropriate, despite possible reciprocal changes in glycogen breakdown compared with gluconeogenesis. Reciprocation could replenish the glucose-6phosphate pool such that a reduction in gluconeogenesis might lower glucose-6-phosphate and reflexively increase liver glycogen breakdown. The rate of glucose appearance would be regulated, however, by the final enzyme in the production pathway. There is recent evidence that glucose-6-phosphatase changes rapidly during insulin infusion [47]. It is tempting to speculate that insulin, or possibly NEFA, control glucose-6-phosphatase on a moment-to-moment basis and thus regulate EGP independent of individual changes in either gluconeogenesis or glycogenolysis. Whether modulation of glucose-6-phosphatase is a primary mechanism for overall regulation of EGP still requires careful examination.

\section{Why is insulin secreted into the portal vein?}

If we can assume that much of the control of liver glucose output is extrahepatic and that NEFA can exert this control, it is of importance to ask "why has evolution chosen for insulin to be secreted into the portal vein?" If the direct effect of insulin on the liver is not a dominant mechanism for EGP control, then the beta cells could have released insulin just as well into the general circulation. Although it is never possible to answer such a teleologic question with certainty, teleology is an important tool in understanding the underlying "design principles" which have led to mammalian organisms as we observe them. Recent evidence does allow for speculation regarding why there could have been survival advantage for secretion of insulin in the portal vein, rather than in the systemic circulation.

We have recently examined the time-dependent effects of moderate fat feeding in the conscious normal dog model [48]. In our experiments, a modest fat diet was used $(+50 \mathrm{~g} /$ day $)$ which often did not result in net weight gain but increased central adiposity by about $50 \%$ in 8 weeks. As expected, the increase in visceral fat was associated with insulin resistance; insulin sensitivity was reduced approximately $75 \%$. What were the physiological responses to this omental fat deposit? As previously predicted $[49,50]$, beta-cell response to glucose was enhanced to compensate for insulin resistance such that glucose tolerance was not reduced in the dog model for at least 6 weeks. Whereas the first-phase beta-cell response was maximised after 6 weeks of fat feeding, after that the increase in islet-cell (first-phase) sensitivity to glucose was partially reversed, even though glucose tolerance remained in the normal range. Beta-cell hyperfunction was relieved by a $50 \%$ reduction in insulin clearance, presumably due to reduced first-pass hepatic insulin extraction (Fig. 2). Although the signal for reduced liver insulin extraction is not known, it could well be portal NEFA. They have been shown to reduce insulin clearance in vitro [51] and in vivo [52]. Thus, with changes in central fat stores, which causes peripheral insulin resistance $[53,54]$, one response is a compensatory reduction in liver insulin extraction, allowing for a greater proportion of secreted insulin to enter the systemic circulation. Thus, we suggest that insulin is secreted into the portal vein not only to allow for direct moment-to-moment control of the liver by insulin but also, to allow the liver to be a gatekeeper for secreted insulin: under insulin-sensitive conditions insulin can be degraded rapidly by liver because limited amounts of the hormone are needed by the insulin-sensitive tissues (muscle and adipose tissue). Under insulin resistant conditions, islet function up-regulates initially; the reduction in clearance of insulin by the liver secondarily shuttles a greater percentage of secreted insulin to the general circulation, sparing the islet cells from oversecreting to maintain normal carbohydrate and lipid metabolism. By this scenario, pancreatic islets secrete insulin into the portal vein to allow the liver to act as a gatekeeper for insulin, reducing the longterm need for insulin oversecretion by the pancreatic islets, and possibly, in the long term, reducing or preventing beta-cell stress and ultimately beta-cell failure. Whether failure of this liver gatekeeper mechanism plays a part in the pathogenesis of Type II diabetes is still to be investigated.

It is clear that investigation of the principles which determine liver glucose output, a fundamental process essential to life, has and will continue to show important insights into the normal and abnormal holistic functioning of the mammalian organism.

Acknowledgements. We appreciate the efforts of Dr. M. Ader in preparation of this manuscript. Dr. Bergman is supported by the National Institutes of Health (USA); grants DK27 619 and DK29867. 


\section{References}

1. Martin BC, Warram JH, Krolewski AS, Bergman RN, Soeldner JS, Kahn CR (1992) Role of glucose and insulin resistance in development of type 2 diabetes mellitus: results of a 25-year follow-up study. Lancet 340: 925-929

2. Mykkanen L, Haffner SM, Ronnemaa T, Bergman R, Laakso M (1997) Low insulin sensitivity is associated with clustering of cardiovascular risk factors. Am J Epidemiol 146: $315-321$

3. Modan M, Halkin H, Almog S et al. (1985) Hyperinsulinemia: a link between hypertension, obesity, and glucose intolerance. J Clin Invest 75: 809-817

4. Giovannucci E (1995) Insulin and colon cancer. Cancer Causes Control 6: 164-179

5. Reaven GM (1988) Role of insulin resistance in human disease (Banting Lecture). Diabetes 37: 1595-1607

6. Saltiel AR, Olefsky JM (1996) Thiazolidinediones in the treatment of insulin resistance and Type II diabetes. Diabetes 45: 1661-1669

7. Berkowitz K, Peters R, Kjos SL et al. (1996) Effect of troglitazone on insulin sensitivity and pancreatic $\beta$-cell function in women at high risk for NIDDM. Diabetes 45: 1572-1579

8. Martin G, Schoonjans K, Staels B, Auwerx J (1998) PPAR$\gamma$ activators improve glucose homeostasis by stimulating fatty acid uptake in the adipocytes. Atherosclerosis 137: S75-S80

9. McGarry JD, Dobbins RL (1999) Fatty acids, lipotoxicity and insulin secretion. Diabetologia 42: 128-138

10. Dresner A, Laurent D, Marcucci M et al. (1999) Effects of free fatty acids on glucose transport and IRS-1-associated phosphatidylinositol 3-kinase activity. J Clin Invest 103: 253-259

11. Roden M, Price TB, Perseghin G et al. (1996) Mechanism of free fatty acid-induced insulin resistance in humans. $\mathrm{J}$ Clin Invest 97: 2859-2865

12. Ganong WF (1987) Review of Medical Physiology, 13th edn. Appleton and Lange, East Norwalk

13. Rizza RA, Mandarino LJ, Gerich JE (1981) Dose-response characteristics for effects of insulin on production and utilization of glucose in man. Am J Physiol 240: E630-E639

14. Hems DA, Whitton PD (1980) Control of hepatic glycogenolysis. Physiol Rev 60: 1-50

15. Hers HG, Hue L (1983) Gluconeogenesis and related aspects of glycolysis. Annu Rev Biochem 52: 617-653

16. Levine R, Fritz IB (1956) The relation of insulin to liver metabolism. Diabetes 5: 209-219

17. Bradley DC, Poulin RA, Bergman RN (1992) Dynamics of hepatic and peripheral insulin effects suggest common ratelimiting step in vivo. Diabetes 42: 296-306

18. Pigon J, Giacca A, Ostenson CG, Lam L, Vranic M, Efendic S (1996) Normal hepatic insulin sensitivity in lean, mild noninsulin-dependent diabetic patients. J Clin Endocrinol Metab 81: 3702-3708

19. Rebrin K, Steil GM, Getty L, Bergman RN (1995) Free fatty acid as a link in the regulation of hepatic glucose output by peripheral insulin. Diabetes 44: 1038-1045

20. Prager R, Wallace P, Olefsky JM (1986) In vivo kinetics of insulin action on peripheral glucose disposal and hepatic glucose output in normal and obese subjects. J Clin Invest 78: 472-481

21. Finegood DT, Bergman RN, Vranic M (1987) Estimation of endogenous glucose production during hyperinsulinemiceuglycemic glucose clamps: comparison of unlabeled and labeled exogenous glucose infusates. Diabetes 36: 914-924

22. Bell PM, Firth RG, Rizza RA (1986) Assessment of insulin action in insulin-dependent diabetes mellitus using $\left[6-{ }^{14} \mathrm{C}\right]-$ glucose, $\left[3-{ }^{3} \mathrm{H}\right]$-glucose, and $\left[2-{ }^{3} \mathrm{H}\right]$-glucose: differences in the apparent pattern of insulin resistance depending on the isotope used. J Clin Invest 78: 1479-1486

23. Molina JM, Baron AD, Edelman SV, Brechtel G, Wallace P, Olefsky JM (1990) Use of a variable tracer infusion method to determine glucose turnover in humans. Am J Physiol 258: E16-E23

24. Finegood DT, Bergman RN, Vranic M (1988) Modelling error and apparent isotope discrimination confound estimation of endogenous glucose production during euglycemic glucose clamps. Diabetes 37: 1025-1034

25. McMahon MM, Schwenk WF, Haymond MW, Rizza RA (1989) Underestimation of glucose turnover measured with $\left[6-{ }^{3} \mathrm{H}\right]-$ and $\left[6,6-{ }^{2} \mathrm{H}\right]-$ but not $\left[6-{ }^{14} \mathrm{C}\right]$ glucose during hyperinsulinemia in humans. Diabetes 38: 97-107

26. Turk D, Alzaid A, Dinneen S, Nair KS, Rizza R (1995) The effects of non-insulin-dependent diabetes mellitus on the kinetics of onset of insulin action in hepatic and extrahepatic tissues. J Clin Invest 95: 755-762

27. Jensen I, Kruse V, Larsen UD (1991) Scintigraphic studies in rats: kinetics of insulin analogues covering wide range of receptor affinities. Diabetes 40: 628-632

28. Bergman RN (1997) New concepts in extracellular signaling for insulin action: the single gateway hypothesis. Recent Prog Horm Res 42: 359-385

29. Ader M, Bergman RN (1990) Peripheral effects of insulin dominate suppression of fasting hepatic glucose production. Am J Physiol 258: E1020-E1032

30. Rebrin K, Steil GM, Mittelman S, Bergman RN (1996) Causal linkage between insulin regulation of lipolysis and liver glucose output. J Clin Invest 98: 741-749

31. Mittelman SD, Fu YH, Rebrin K, Steil G, Bergman RN (1997) Indirect effect of insulin to suppress endogenous glucose production is dominant, even with hyperglucagonemia. J Clin Invest 100: 3121-3130

32. Giacca A, Fisher S, Shi ZQ, Gupta R, Lickley HL, Vranic M (1992) Importance of peripheral insulin levels for insulin-induced suppression of glucose production in depancreatized dogs. J Clin Invest 90: 1769-1777

33. Lewis GF, Zinman B, Groenewoud Y, Vranic M, Steiner G, Giacca A (1996) Hepatic glucose production is regulated both by direct hepatic and extrahepatic effects of insulin in humans. Diabetes 45: 454-462

34. Lewis GF, Vranic M, Harley P, Giacca A (1997) Fatty acids mediate the acute extrahepatic effects of insulin on hepatic glucose production in humans. Diabetes 46: 1111-1119

35. Lewis GF, Vranic M, Giacca A (1997) Glucagon enhances the direct suppressive effect of insulin on hepatic glucose production in humans. Am J Physiol 272: E371-E378

36. Lewis GF, Carpentier A, Vranic M, Giacca A (1999) Resistance to insulin's acute direct hepatic effect in suppressing steady-state glucose production in individuals with Type 2 diabetes. Diabetes 48: 570-576

37. Exton JH, Corbin JG, Park CR (1969) Control of gluconeogenesis in liver: IV. Differential effects of fatty acids and glucagon on ketogenesis and gluconeogenesis in the perfused rat liver. J Biol Chem 244: 4095-4102

38. O'Meara NM, Blackman JD, Sturis J, Polonsky KS (1993) Alterations in the kinetics of C-peptide and insulin secretion in hyperthyroidism. J Clin Endocrinol Metab 76: 79-84

39. Glinsmann WH, Hern EP, Lynch A (1969) Intrinsic regulation of glucose output by rat liver. Am J Physiol 216: 698-703

40. Bell PM, Firth RG, Rizza RA (1986) Effects of hyperglycemia on glucose production and utilization in humans: measurement with $\left[2^{3} \mathrm{H}\right]-,\left[3^{3} \mathrm{H}\right]-$, and $\left[6^{14} \mathrm{C}\right]$ glucose. Diabetes 35: 642-648 
41. Best JD, Kahn SE, Ader M, Watanabe RM, Ni T-C, Bergman RN (1996) Role of glucose effectiveness in the determination of glucose tolerance. Diabetes Care 19: 1018-1030

42. Mittelman SD, Bergman RN (2000) Inhibition of lipolysis causes suppression of endogenous glucose production independent of changes in insulin. Am J Physiol (in press)

43. Chen X, Iqbal N, Boden G (1999) The effects of free fatty acids on gluconeogenesis and glycogenolysis in normal subjects. J Clin Invest 103: 365-372

44. Clore JN, Glickman PS, Helm ST, Nestler JE, Blackard WG (1991) Evidence for dual control mechanism regulating hepatic glucose output in nondiabetic men. Diabetes 40: $1033-1040$

45. Puhakainen I, Koivisto VA, Yki-Jarvinen H (1991) No reduction in total hepatic glucose output by inhibition of gluconeogenesis with ethanol in NIDDM patients. Diabetes 40: $1319-1327$

46. Massillon D, Chen W, Barzilai N et al. (1998) Carbon flux via the pentose phosphate pathway regulates the hepatic expression of the glucose-6-phosphatase and phosphoenolpyruvate carboxykinase genes in conscious rats. J Biol Chem 273: 228-234

47. Dea MK, Kim SP, Kabir M, Youn JH, Bergman RN (2000) Insulin suppresses glucose production by inhibiting glucose-6-phosphatase. Diabetes 49 [Suppl 1]: A282
48. Mittelman SD, van Citters GW, Kim SP, Davis DA, Dea MK, Bergman RN (2000) The longitudinal compensation for fat induced insulin resistance includes reduced insulin clearance and enhanced B-cell response. Diabetes (in press)

49. Bergman RN, Phillips LS, Cobelli C (1981) Physiologic evaluation of factors controlling glucose tolerance in man: measurement of insulin sensitivity and B-cell glucose sensitivity from the response to intravenous glucose. J Clin Invest 68 : $1456-1467$

50. Bergman RN (1989) Toward physiological understanding of glucose tolerance: minimal-model approach (Lilly Lecture). Diabetes 38: 1512-1527

51. Svedberg J, Stromblad G, Wirth A, Smith U, Bjorntorp P (1991) Fatty acids in the portal vein of the rat regulate hepatic insulin clearance. J Clin Invest 88: 2054-2058

52. Wiesenthal SR, Sandhu H, McCall RH et al. (1999) Free fatty acids impair hepatic insulin extraction in vivo. Diabetes 48: 766-774

53. Arner P, Pollare T, Lithell H (1991) Different aetiologies of Type 2 (non-insulin-dependent) diabetes mellitus in obese and non-obese subjects. Diabetologia 34: 483-487

54. Ruderman N, Chisholm D, Pi-Sunyer X, Schneider U (1998) The metabolically obese, normal-weight individual revisited. Diabetes 47: 699-713 